\title{
Identification of key regulators in glycogen utilization in E. coli based on the simulations from a hybrid functional Petri net model
}

\author{
Zhongyuan Tian', Adrien Fauré ${ }^{1}$, Hirotada Mori², Hiroshi Matsuno ${ }^{*}$ \\ From 24th International Conference on Genome Informatics (GIW 2013) \\ Singapore, Singapore. 16-18 December 2013
}

\begin{abstract}
Background: Glycogen and glucose are two sugar sources available during the lag phase of E. coli, but the mechanism that regulates their utilization is still unclear.

Methods: Attempting to unveil the relationship between glucose and glycogen, we propose an integrated hybrid functional Petri net (HFPN) model including glycolysis, PTS, glycogen metabolic pathway, and their internal regulatory systems.

Results and conclusions: By comparing known biological results to this model, basic necessary regulatory mechanism for utilizing glucose and glycogen were identified as a feedback circuit in which HPr and EllA ${ }^{\text {Glc }}$ play key roles. Based on this regulatory HFPN model, we discuss the process of glycogen utilization in $E$. coli in the context of a systematic understanding of carbohydrate metabolism.
\end{abstract}

\section{Background}

The carbohydrate pathway occupies a central position in a cell's metabolism. In our previous paper [1], we proved that glycogen plays an important role in the lag phase of $E$. coli. But how the cell regulates the utilization of these carbon sources, intracellular glycogen and extracellular glucose, was yet to be clarified. In a cell, glycogen works as a sugar store or a sugar supply depending on different nutrition conditions, under the regulation of enzymes expressed by $g l g$ gene clusters $(g \lg B X C A P)$ [2]. Uptake of extracellular glucose is conducted via the phosphotransferase system (PTS) in E. coli, whose enzymes are expressed from two operons, ptsHIcrr and ptsG [3]. Although several shared regulators of PTS and glycogen metabolism, such as ppGpp, Cra, CsrA and cAMP/CRP, have been studied [2,4-10], a basic regulation system for the utilization of glucose and glycogen has not been studied yet.

\footnotetext{
* Correspondence: hmatsuno@yamaguchi-u.ac.jp

'Graduate School of Science and Engineering, Yamaguchi University, 1677-1 Yoshida, 753-8512 Yamaguchi-shi, Yamaguchi, Japan

Full list of author information is available at the end of the article
}

Computer modeling is a general and effective method for the integration of biological systems. In our previous work [1], at first we calculated glucose and G6P concentrations, from which we predicted the existence of another major sugar donor, glycogen, in the lag phase. The function of glycogen as a sugar donor was simulated, and demonstrated experimentally. The purpose of this paper is to construct an integrated model for the systematic understanding of the carbohydrate pathway system of E. coli. In this work we firstly constructed two hybrid functional Petri net (HFPN) models [11] based on two types of published models: ordinary differential equation (ODE) model of central carbohydrate pathway [12] and a mass balance theory model of PTS [13]. These two models were then assembled together with a newly developed general mass action model of the glycogen metabolic pathway into a single, comprehensive HFPN model.

By applying metabolic regulatory mechanisms in our combined HFPN model, a basic control system regulating the utilization glucose and glycogen was identified, in which HPr::GlgP complex [14-16], EIIA ${ }^{\text {Glc } \& \text { cAMP }}$ system [8,17], EI dimerization [18,19], FDP\&Cra mutual 
feedback [6], HPr subcellular location [2,16,20] etc. are working as regulators. In this paper, with the support of simulation results from the HFPN model, we clarify functions of HPr and EIIA ${ }^{\text {Glc }}$ as key regulators of glucose and glycogen utilization.

\section{Results}

Molecular mechanisms for regulating glucose and glycogen utilization

There are many regulators working on central metabolism of $E$. coli [2,4-10]. Among them we selected basic regulators that control glucose and glycogen utilization, which is illustrated in Figure 1A using the molecular interaction map notation [21], a widely recognized standard notation capable of describing biological regulatory networks in a way of electronic circuit. These basic regulators constitute a circuit that gives a whole view of the regulation of glucose and glycogen utilization as showing in Figure 1B. These components are classified into 3 regulation pathways (HPr phosphorylation regulatory pathway (includes actions labeled $S \mathbf{1}, S 2, S 3, P 1, S 4), \mathrm{HPr}$ localization regulatory pathway (includes actions labeled $S 1, S 2, S 3, L 1, L 2, S 4$ ), and gene expression regulatory pathway in PEIIA ${ }^{\text {Glc }}$ \&CAMP subpath-way (includes actions labeled $S 1, S 2, S 3, G 1.1, G 1.2, G 3)$, and in FDP\&Cra subpathway (includes actions labeled G2.1, G2.2, G3, G4)) "Shared regulatory pathway" denotes shared pathways of aforementioned 3 pathways. This diagram is constituted by 5 level hierarchies of metabolite level (M-level), molecule localization level (L-level), phosphate flux level (F-level), protein level(P-level), gene expression level (G-level).

\section{HPr phosphorylation regulatory pathway}

Transition of phosphorylation states of HPr is in charge of the functions of PTS and GlgP [2,8], which occupying a key role at regulation of glucose and glycogen utilization. This HPr centered self feedback control pathway starts from PEP concentration (M-level), terminates at glycogen decomposition (M-level).

Regulations in this pathway includes actions labeled $\boldsymbol{S 1}$, $S 2, S 3, P 1, S 4$. Regulation S1 (from M-level to P-level): high enough PEP levels activate the phosphate group influx into PTS by stimulating EI dimerization $[18,19]$. Regulation $\boldsymbol{S} 2$ (from P-level to F-level): EI dimerization controls phosphate influx into PTS, which is thought to be the limiting step in the process of phosphate group transfering from PEP to G6P via PTS [19]. Regulation $S 3$ (from F-level to F-level): Different phosphorylation states of HPr is resulted from the balance of phosphate group influx to PTS from PEP and outflux to G6P from PTS. Regulation P1 (from F-level to P-level): Different phosphorylation states of HPr result in different phosphorylation states of protein to protein interactions (P)HPr::GlgP (From here on, PHPr denotes the phosphorylated form of
$\mathrm{HPr}, \mathrm{HPr}$ denotes the unphosphorylated form, and (P) HPr denotes both phosphorylated and unphosphorylated Hpr), or (P)EIIA ${ }^{G l c}$ ::(P)HPr::(P)EI (HPr works as a member enzyme of PTS). Regulation $\boldsymbol{S} 4$ (from P-level to M-level): The phosphorylation state of the (P)HPr::GlgP complex controls glycogen decomposition, of which catalyzing speed of HPr::GlgP is about five times higher than that of PHPr::GlgP [14]. In PTS, HPr transfers phosphate group from PEI to EIIA ${ }^{G l c}$. Thus (P)HPr regulates the speed of carbohydrate decompositions from both glycogen and glucose.

\section{HPr localization regulatory pathway}

Lopian et al. (2010) described the spatial and temporal organization of PTS enzymes in E. coli, especially HPr and EI [20]. According to their study, HPr and EI mainly stay in the poles of a cell independently, and if HPr is released to the cytosol, it should be phosphorylated by PEI in the presence of glucose. Genobase also supplies a GFP photo of HPr localization, which is scattered in the cytosol [22]. In the glycogen metabolism, interestingly, glycogenesis enzymes (GlgC, GlgA) and glycogen granules locate at the poles, while GlgP is scattered in the cytosol [2]. GlgP is considered to be always bound in a complex with HPr, since the concentration of HPr is much higher than that of GlgP in E. coli $[14,15]$.

Based on these studies, we hypothesize that HPr controls the priority in glucose and glycogen utilization in E. coli. (1) If there is no glucose, HPr cannot get phosphate from EI, keeping its location at the poles. Hence, this pole-located HPr mainly serves for glycogen decomposition, whose speed is regulated by phosphorylation state of (P)HPr::GlgP. (2) If there is a little glucose supply, at the very beginning of lag phase, glucose uptake takes place at poles areas for a very short time until all the phosphates are removed from these PTS enzymes, including HPr. Note that the pole-located HPr also has the ability of exchanging phosphate with other PTS enzymes. (3) If glucose is abundant, HPr gets phosphate group from PEI, causing its release to the cytosol. Cytosol-scattered HPr works as a PTS protein, but not for glycogenolysis, hence, transporting phosphate from EI to EIIA ${ }^{\text {Glc }}$

Regulations in this pathway includes actions labeled $\boldsymbol{S 1}$, $S 2, S 3, L 1, L 2, S 4$. Regulations $S 1, S 2$, and $S 4$ are the same as those in phosphate flux regulatory pathway. Regulation $\boldsymbol{S} 3$ (from F-level to F-level): The flux of phosphate group into PTS from PEP influences the flux of phosphate group from PEI to PHPr. Regulation $\mathbf{L} 1$ (from F-level to L-level): When there are phosphate groups flux from PEI to PHPr, PHPr will be translocated from poles to the whole cytosol [20]. Regulation $\boldsymbol{L} 2$ (from L-level to P-level): When (P)HPr is located at the cell's poles, it mainly functions for glycogen phosphorylation. And when (P)HPr is scattered in cytosol, it serves for the 


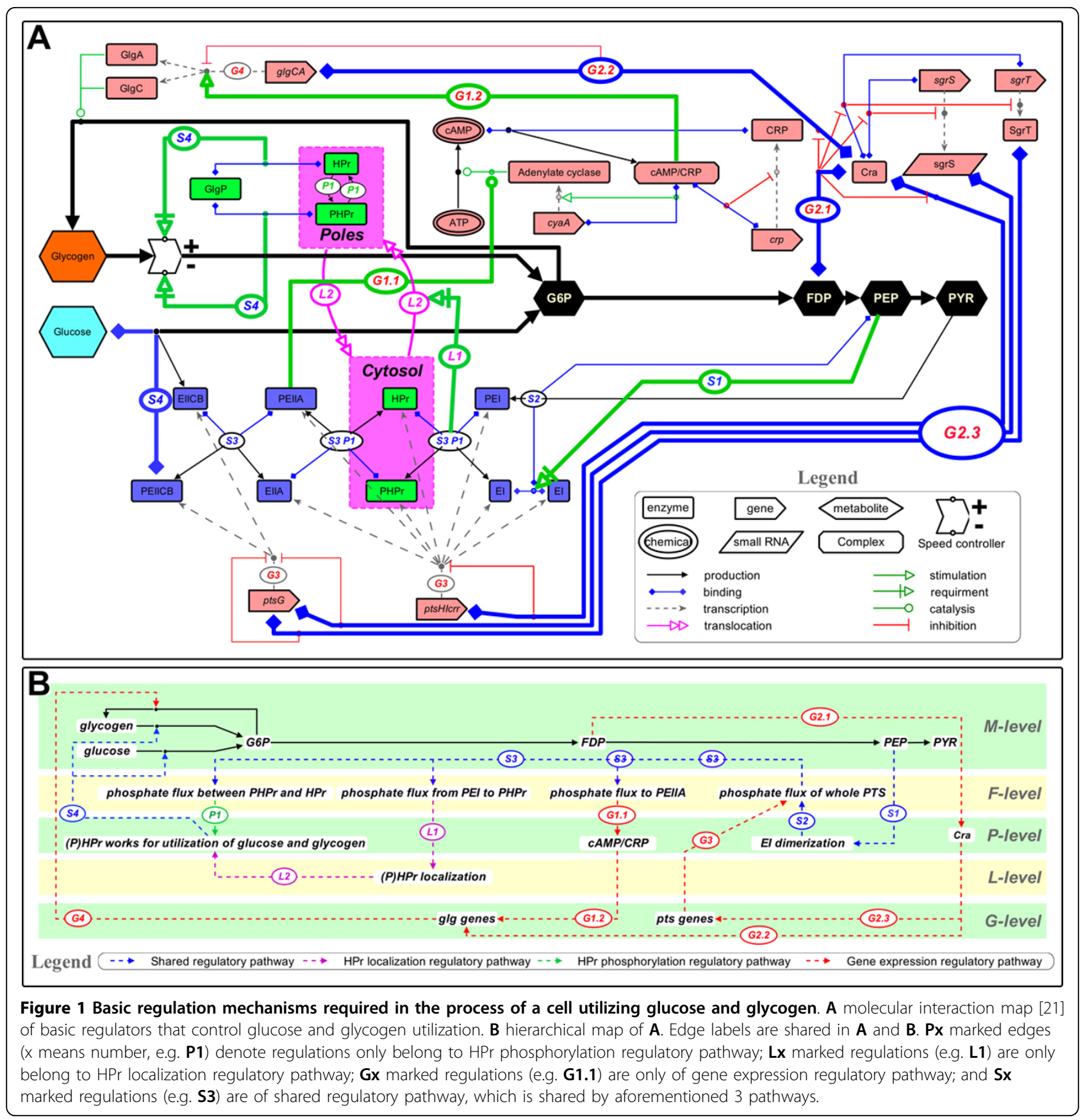

function of PTS, which is responsible for glucose uptake. At last this pathway goes back to regulation $\boldsymbol{S 4}$ to finish its regulation.

\section{Gene expression regulatory pathway}

This pathway explains how an E. coli controls glycogen and glucose utilization in gene expression level. PTS enzymes for glucose uptake in E. coli include EI, HPr, EIIA ${ }^{\text {Glc }}$ and $\mathrm{EIICB}^{G l c}$, in which the former three enzymes are expressed from $p t s H I-c r r$ gene cluster and $\mathrm{EIICB}^{\text {Glc }}$ is from ptsG. Cra is known as a global DNA-binding regulator of the genes for carbon metabolism in $E$. coli, which directly regulates ptsHIcrr operon [6], and indirectly influences ptsG transcription via SgrT and small RNA SgrS pathway $[6,23]$. Fructose-1,6-bisphosphate (FDP) inhibits free Cra level by binding its gene [6]. In E. coli, clustering methods of glycogen associated genes are complex and discussable, such as $g \lg B X C A P, g \lg B X, g \lg C A P, g \lg A P$ etc. $[2,17,24]$, in which GlgC and GlgA catalyzed glycogen synthesis have been experimentally proved to be regulated by cAMP/CRP and Cra $[6,17]$. Gene expression regulatory pathway of this 
study includes two subpathways, PEIIA ${ }^{\text {Glc }}$ \&cAMP subpathway and FDP\&Cra subpathway.

In PEIIA ${ }^{G l c}$ \&CAMP subpathway (includes actions labeled S1, S2, S3, G1.1, G1.2, G3), regulations $S 1$ and $S 2$ are shared with the other two regulation path-ways. Regulation $\boldsymbol{S} 3$ (from F-level to F-level): The flux of phosphate group into PTS from PEP influences the phosphate intraflux between EIIA ${ }^{G l c}$ and PEIIA ${ }^{G l c}$. Regulation G1.1 (F-level to P-level): The phosphate flux between EIIA ${ }^{\text {Glc }}$ and PEIIA ${ }^{\text {Glc }}$ controls intracellular PEIIA ${ }^{\text {Glc }}$ concentration, which stimulates adenylate cyclase (AC) to produce much cAMP. In Figure 1A, we can see a local feedback loop constituted by cAMP, CRP and cAMP/CRP, the binding complex of them $[7,8,17]$. Regulation $\mathbf{G 1 . 2}$ (P-level to G-level): cAMP/CRP network upregulating $g \lg C$ and $g \lg A$ expression is confirmed by experiments of [17]. Because expressions of $g \lg C$ and $g \lg A$ (Regulation G4) are under combined regulation of PEIIA ${ }^{\text {Glc }}$ \&cAMP subpathway and FDP\&Cra subpathway, we will explain it later in the following subpathway.

In FDP\&Cra subpathway (includes actions labeled G2.1, G2.2, G3, G4), regulations G2.1 (M-level to P-level) and G2.2 (P-level to G-level): When FDP reaches a high level, Cra expression is repressed, which releases its regulations on $g l g C$, glgA, ptsHIcrr directly and $p t s G$ via SgrST route [6]. Regulation G3 (G-level to F-level): After an exponential increasing, when an enzyme concentration increases above a certain threshold, its catalyzed reaction speed will remain in a high level [25]. Here we assumed that, when PTS enzymes are expressed above a certain threshold, the whole PTS reaction speed would be extremely accelerated. Regulation G4 (G-level to M-level): $g l g C$ and $g l g A$ expression levels are under regula-

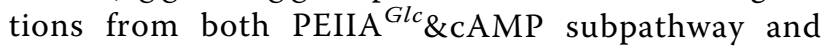
FDP\&Cra subpathway. Comprehensively say, when Cra levels decreases, it releases the inhibition of $g l g C$ and $g l g A$, as a consequence cAMP/CRP activates extremely strong expression of $g \lg C$ and $g \lg A$ (Details are discussed in Results section).

Construction of a dynamic simulation model of central metabolic pathway with HFPN Central metabolic pathway in E. coli is constituted by the glycolysis, the pentose phosphate (PP) pathway, and the tricarboxylic acid cycle (TCA cycle). Most glycolysis models are based on ODE [12,26,27]. Chassagnole et al. (2002) constructed an integrated ODE model of glycolysis and PP pathways [12,28], which is often used as a base model in many studies $[26,27,29,30]$. By assembling TCA cycle with the model of [12], Kadir et al. (2010) set up an ODE model together with six pieces of logical controlling rules [27], and Usuda et al. (2010) included gene regulation in [26]. Kinetic parameters of these ODE model were taken from the databases, such as BRENDA [31], SABIO-RK [32], and BioModels [33], and some works focused on parameter optimization [30]. PTS are usually represented by one or a few equations in these ODE models. Rohwer at el (2000) set a mass balance theory model of PTS, by using experimentally tested mass action constant for each elementary biochemical reaction within PTS enzymes [13], and some studies are based on it $[9,34]$.

The simulation of our HFPN models are conducted on Cell Illustrator 4.0 [35]. Before realizing a whole model, we have first set up two independent HFPN models based on these published ODE models of glycolysis and PP pathway [12,33] (see Additional file 1: Model-1 [36], HFPN models of this work (Mode-1 in Additional file 1, Mode-2 in Additional file 2, Mode-3 in Additional file 3 and Mode-4 in Additional file 4) and their simulation results (Figure $\mathrm{S}$ in Additional file 5), approaches (Methods in Additional file 6) and parameters (Table $\mathrm{S}$ in Additional file 7) are supplied in URL [36].) and mass balance theory models of PTS [13,34] (see Additional file 2: Model-2 [36]). Subsequently, these two HFPN models are combined into one (see Additional file 3: Model-3 [36]). This combined HFPN model was further extended by incorporating glycogen metabolism pathway and basic regulatory mechanisms, and finally we got an extended HFPN model of carbohydrate metabolism, as shown in Figure 2. We employed general mass action method to construct this integrated HFPN model (see Additional file 4: Model-4 [36]), in which mass action constants were manually fitted so as to meet biological data of glycogen and other metabolites concentrations from our former study [1]. For example from G6P to G1P to glycogen via ADPG, we obtained their mass action factors by adjusting the model's behavior to the biological data: that is, mass action parameters were determined based on the known information of concentrations of reactants and products. In order to check the availability of our integrated model (Model-4, see Additional file 4[36]), we made a comparison between the results from this model and from the model of glycolysis and PP pathway [12], showing the consistency of these two models (Figure S1, see Additional file 5[36]).

The integrated HFPN model produced the correct behavior of metabolite concentrations of G6P, PEP, FDP etc. in a batch culture as well as the concentrations of glycogen and extracellular glucose in Figure 3, which can be confirmed by comparing with their experimental data in Supplementary data of [1]. Further, PTS enzymes level are also illustrated in Figure 3. In order to evaluating these simulation results, we calculated their Pearson product-moment correlation coefficient values (r-value) [37] against their experimental data (Figure S2 in Additional file 5[36]). Fortunately, r-valure shows simulation results of important metabolites for this study (G6P, F6P, FDP) are reliable $(|\mathrm{r}|>0.8)$. Those metabolites of $|\mathrm{r}|<0.8$ should 


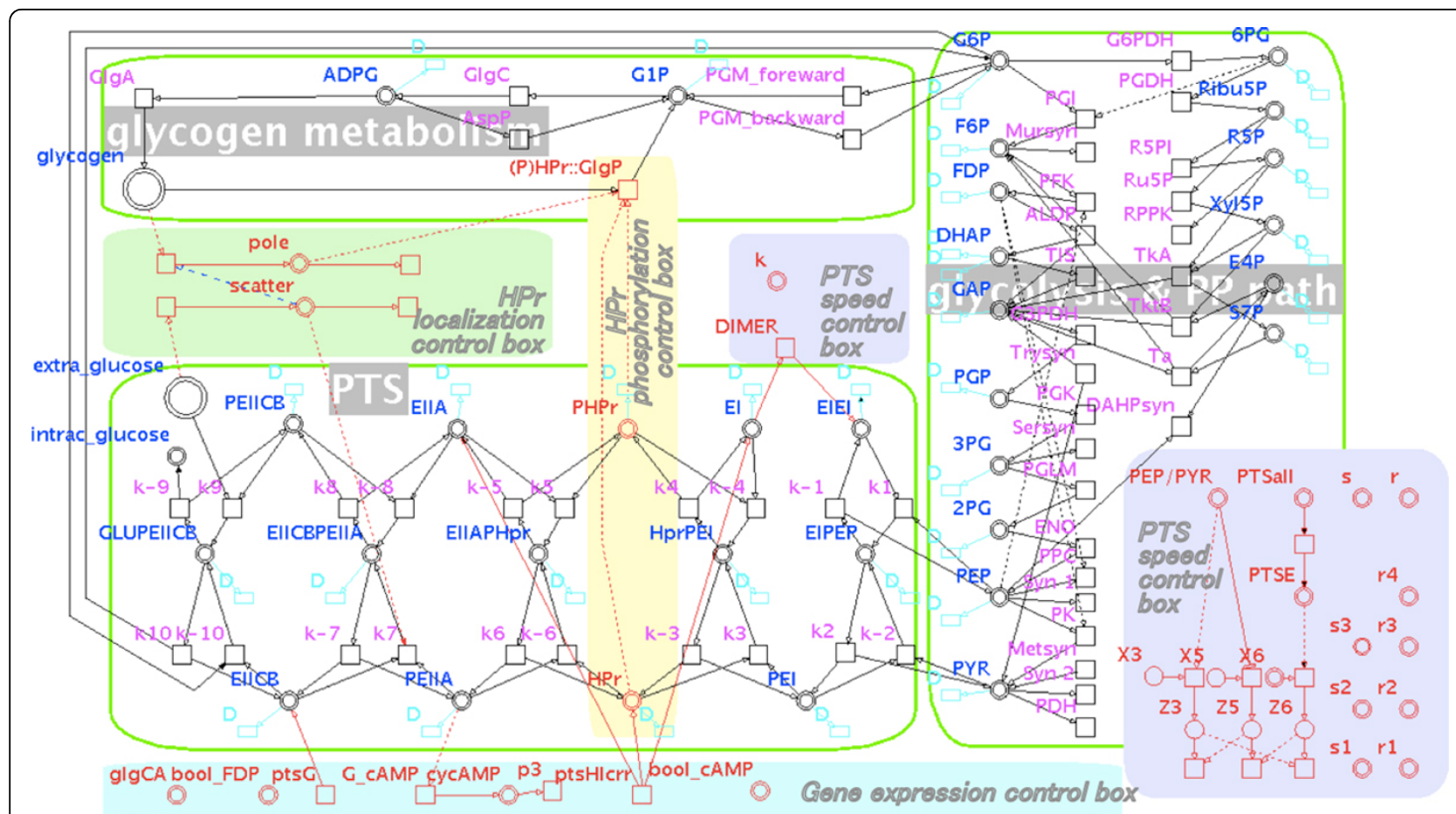

Figure 2 A HFPN model of extended center metabolism pathway in E. coli. This model includes three major parts: glycolysis and pentose phosphate pathway part, PTS part and glycogen metabolism pathway part, and they are bordered by light green lines respectively. Cyan border and D marked transitions are degradation or dilution processes of their connected metabolites or enzymes. Black border components are of the three major parts. Red border components are of regulatory mechanisms, in which transition pts $G$ and ptsH/crr represent PTS genes expression process; G_CAMP is the process of PEIIA ${ }^{\text {Glc }}$ activating CAMP production; Dimer is the process of El dimerization; $\mathbf{k}$ is the parameter controlling the whole PTS reaction speed; Location is of the molecular subcellular localization regulation mechanism; (P)HPr::GlgP represent the process of the binding of (P)HPr::GlgP catalyzing glycogen decomposition. This is the snapshot of main part of our HFPN model, other components can be found in Additional files in URL [36].

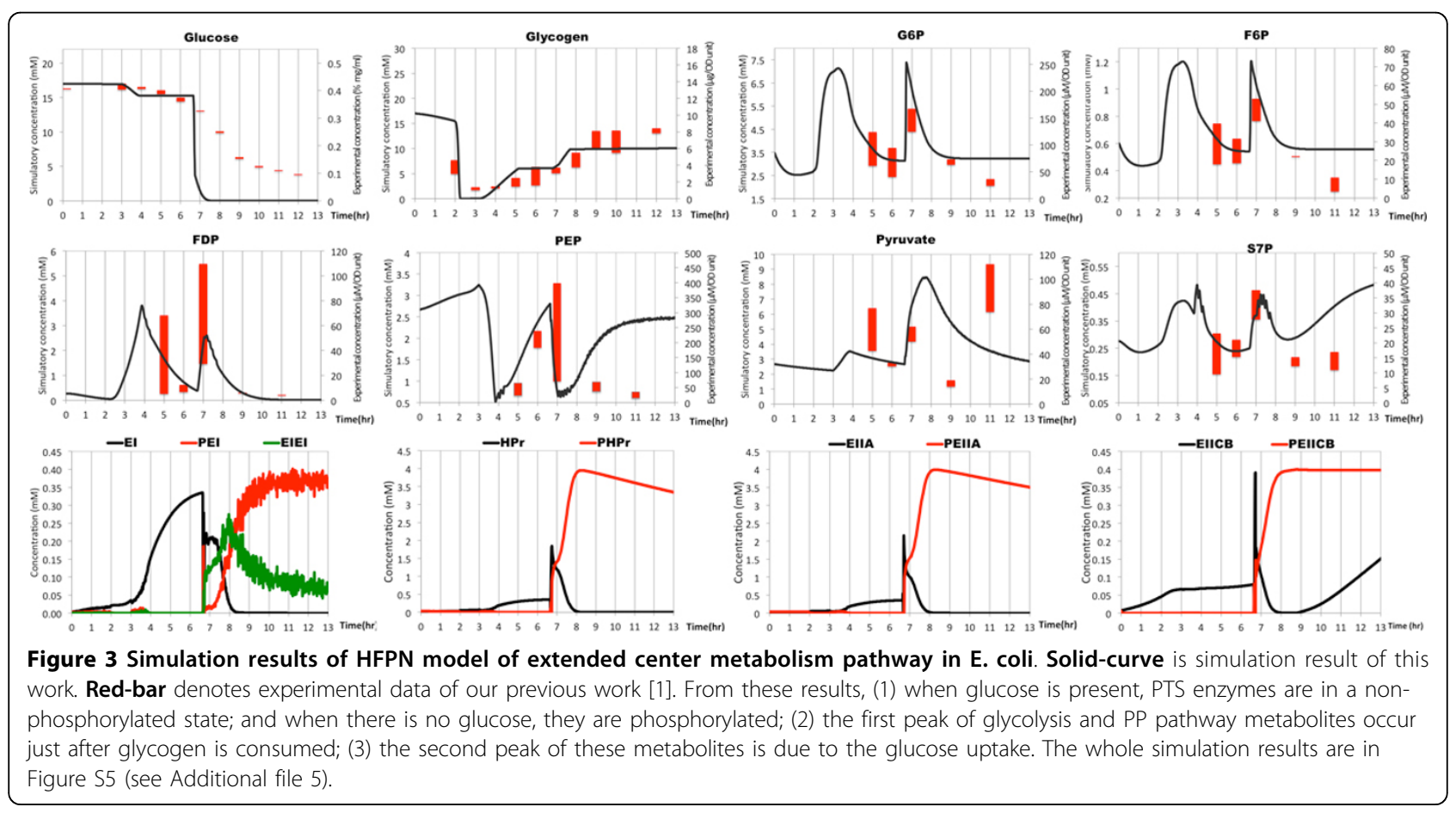


be addressed in the future, their low r-value may come from the lack of other routes connecting to PEP and PYR (for example, acetate pathway, gluconeogenesis etc.), or may come from the parameters of PTS part, which were conducted in vitro [13].

\section{Confirmation of the role of $\mathrm{HPr}$ and ElIA ${ }^{\text {Glc }}$ as key regulators by simulation \\ Biological analyses based on the simulation results}

With running simulations on the constructed HFPN model, we are able to systematically understand the process of carbohydrate metabolism in a batch culture in E. coli along its lifetime, which consists of 5 phases, early lag phase, late lag phase, early log phase, late log phase, and stationary phase (Figure 4B). Simulated concentrations of glucose, glycogen, FDP, HPr (EIIA ${ }^{\text {Glc }}$ ), PHPr (PEIIA ${ }^{\text {Glc }}$ ), cAMP, $g l g C A$, and (P)HPr subcellular localization are shown in Figure 4A.

Early lag phase (1). At the beginning of this phase, $E$. coli begins its growth just after being put into a fresh medium. At this point, (P)HPr is mainly present at the poles and causes a little glucose uptake locally. Glycogen is not utilized well in this phase, because it is surrounded by PHPr. Indeed the higher affinity of PHPr than HPr isolates GlgP from glycogen, resulting in a very slow speed decomposition rate of glycogen.

Early lag phase (2). Although this phase begins with $\mathrm{PHPr}$, this protein slowly loses its phosphate. Because glycolytic pathway is not working in this phase, so phosphate cannot be provided through PTS. As HPr dephosphorylation completes, glycogen catalysis by HPr::GlgP begins, and $E$. coli uses glycogen as its main carbon source. Along with the quick consumption of glycogen, $\mathrm{HPr}$ is moved to the cytosol by the function of PEI [20]. Meanwhile, glycogen supplied phosphate flows into the central metabolic pathway, causing PEP accumulation. Distribution of (P)HPr in the cytosol will be finished at almost the same time.

Late lag phase. This is a period of slow glucose uptake, which is caused by a relevant lower level of PEP, due to a low speed EI dimerization [18]. This means that metabolites produced from glycogen support the transportation of phosphate for glucose uptake. During this period, (P) HPr has been distributed in the cytosol, whose major role is to work for PTS not for glycogen, and this also causes the start of glycogen accumulation. Meanwhile in this phase more PTS enzymes are expressed, preparing for the impending log phase.

Early $\log$ phase. Uptake of glucose is very fast in this phase due to the highly expressed PTS proteins and the active transportation of phosphate by these PTS proteins. Glucose is the main sugar source in this phase.

Late log phase. In this phase, under the combined regulation of PEIIA ${ }^{\text {Glc }}$ (via cAMP/CRP), and FDP (via Cra), $g \lg C$ and $g \lg A$ are expressed at extremely high levels $[2,6,8]$, causing efficient glycogen accumulation. Due to
A

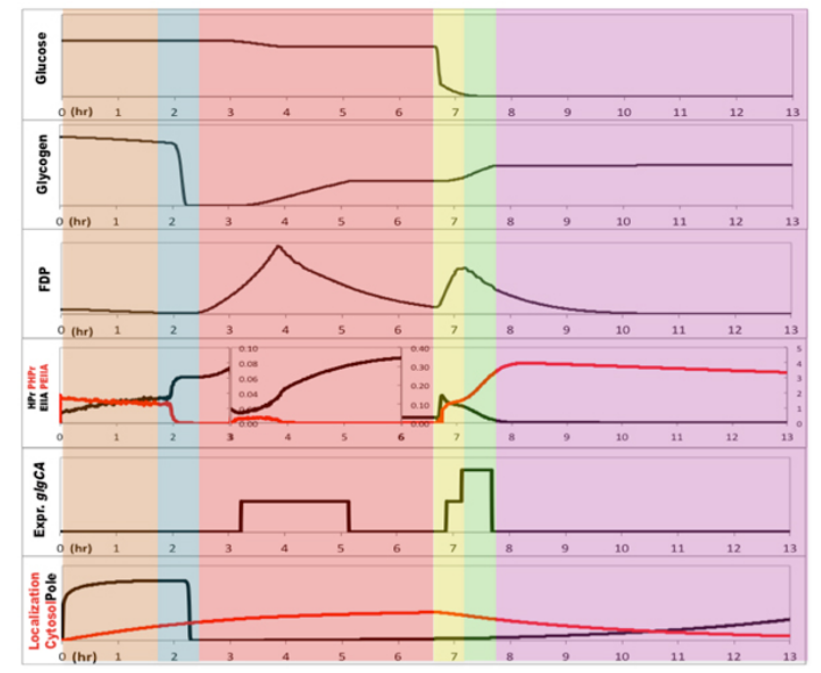

B

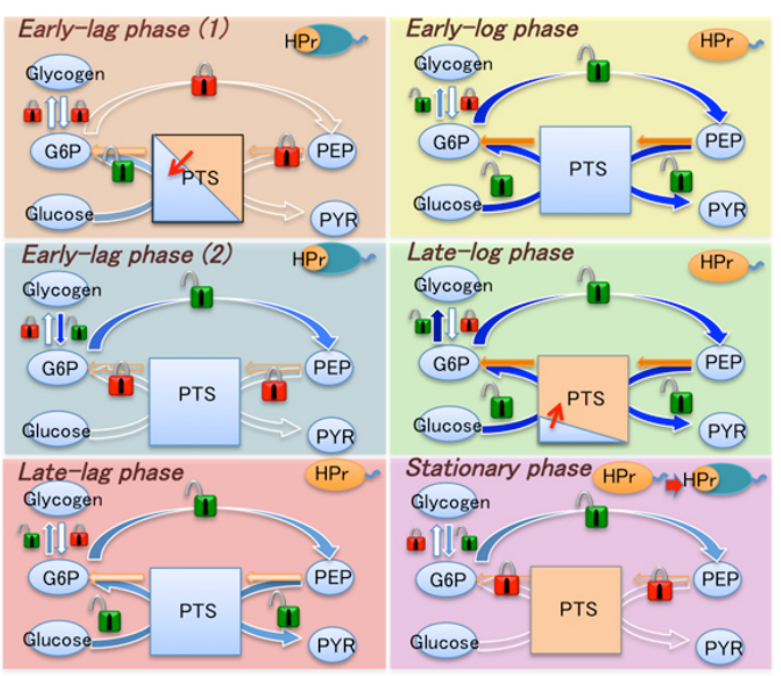

Figure 4 Systematically understanding of the phases of extent center metabolism in an E. coli along its whole lifetime. A illustrates experiment and simulation behaviors of some major metabolites and enzymes, $\mathbf{B}$ shows sugar and phosphate flux processes of $E$. coli utilizing glucose and glycogen. Same color background areas of A and B are of the same phases. In B: Blue colored PTS represents unphosphorylated PTS, orange colored PTS is phosphorylated PTS. Blue filled arrows indicate carbon flux routes, in which deeper blue color represents more flowing amount; orange color filled arrows indicate phosphate flux routes, in which deeper orange color represents more flowing amount. Closed red locks means inactivated pathway; open green locks means activated pathway. The whole orange colored E. coli marked with "HPr" indicates HPr is scattered in cytosol; only orange colored pole E. coli marked with "HPr" indicates HPr is at poles. 
the lower speed of phosphate output from the PTS comparing with its input speed from PEP, high level of PHPr are working for glucose uptake. (P) HPr is mainly expressed in the cytosol, so it can hardly contribute to glycogen decomposition.

Stationary phase. When cells come to a stationary phase, glycogen is in its slow speed catalyzing state. Since (P)HPr is maintained in phosphorylated state, it concentrates towards the poles, where glycogen is located. In the post stationary phase, there is no glucose supplied outside, glycogen is used as a carbon source for cells to survive. Glycogen low speed catalyzation is regulated by surrounding PHPr in poles. Next, if the E. coli is put into another culture, a new lag phase begins.

\section{Qualitative description of regulator states throughout the} phases

Multi-valued formulation. glgC and $g \lg A$ are the genes that forms an operon with $\operatorname{glg} P[2,17,24]$. According to experimental result, $g \lg C$ and $g \lg A$ are regulated by cAMP [2,17] and FDP [6], respectively. Hence we can consider that the transcriptions of these two genes are regulated by the combination of FDP amount and cAMP level, which are distinguished $\alpha(g \lg C \& g \lg A$ activation) and $\beta$ ( $g \lg C \& g \lg A$ activation), respectively. Actually, from the biological literature $[2,6,17]$, it is known that the composition speed of glycogen varies depending on the expression pattern of $\alpha$ and $\beta$. If either $\alpha$ or $\beta$ is expressed, glycogen is composed in slow speed, but if both $\alpha$ and $\beta$ are expressed, glycogen is composed in high speed. This function can be expressed by multi-valued formulation as presented in Table 1 .

Phase transitions based on the regulatory factors. According to aforementioned analysis, the importance of HPr and EIIA ${ }^{\text {Glc }}$ on glycogen regulation is pointed out from a biological point of view. To make it more precise, we will express this regulatory system from an engineering point of view, presenting qualitative description of this system as shown in Table 2. Glycogen process is controlled by the regulators FDP, EIIA ${ }^{\text {Glc }}$, and HPr in the left column of this table. Among them, FDP and EIIA ${ }^{\text {Glc }}$ are involved in glycogen synthesis, and HPr in its decomposition.

Table 1 Multi-valued formulation of the regulation in utilizing glycogen and glucose in $E$. coli.

\begin{tabular}{|c|c|c|}
\hline $\begin{array}{c}\alpha \\
g \lg C \& g \lg A \\
\text { activation }\end{array}$ & $\begin{array}{c}\beta \\
g \lg C \& \text { glgA } \\
\text { activation }\end{array}$ & $\begin{array}{c}\gamma \\
\text { glycogen } \\
\text { composition }\end{array}$ \\
\hline 0 & 0 & 0 \\
\hline 0 & 1 & 1 \\
\hline 1 & 0 & 1 \\
\hline 1 & 1 & 2 \\
\hline
\end{tabular}

$\alpha$ represents $\alpha$ (glgC \& glgA activation), $\beta$ represents $\beta$ (glgC \& glgA activation), $\gamma$ represents $\gamma$ (glycogen composition). The meaning of multi-values are 0 (no/off), 1 (on/slow), and 2 (fast).
In the following, we will show, phase by phase, how composition and decomposition take place on the controls with these regulators in this table.

Early lag phase. Because of "very slow" uptake speed of glucose, FDP amount is in "low" level, resulting in "off" expression of $g l g C \& g l g A$ genes $(\alpha)$. EIIA ${ }^{\text {Glc }}$ and $\mathrm{HPr}$ display the same behavior, changing these phosphorylation states, "yes $\rightarrow$ no". In addition, $g \lg C \& g \lg A$ activation $(\beta)$ is influenced by this state transition as "on $\rightarrow$ off" in Table 2. Glycogen composition, however, is not influenced by these regulations, because the uptake speed of glucose is too slow to produce glycogen. On the other hand, glycogen decomposition takes place in this phase, with changing its speed "slow $\rightarrow$ fast" according to the phosphorylation state transition of HPr "yes $\rightarrow$ no". Hence, glycogen is the major sugar source in this phase.

Late lag phase. Since E. coli has not consumed much energy yet in this phase, FDP accumulates in "high" levels despite the "slow" glucose uptake speed. Hence $g \lg C \& \operatorname{glg} A(\alpha)$ is "on". On the contrary, $\operatorname{glg} C \& \operatorname{glg} A(\beta)$ is "off", which is resulted from "no" phosphorylation state of EIIA ${ }^{\text {Glc }}$ via "low" cAMP level. According to the rule (if $\alpha=1$ and $\beta=0$ then $\gamma=1$ ) in Table 1 , glycogen is composed $(\gamma)$ in "slow" speed. On the other hand, glycogen decomposition does not take place in this phase, because $\mathrm{HPr}$ is not located at the poles but distributed in the cytosol, which does not satisfy the requirement for glycogen decomposition.

Early log phase. Due to "very fast" speed of glucose uptake, FDP is accumulated in E. coli, despite its high metabolic activity, changing its amount as "low $\rightarrow$ high". Accordingly, the state of $g \lg C \& g \lg A(\alpha)$ activation is changed as "off $\rightarrow$ on". In this stage, HPr is not phosphorylated, then the expression of $\operatorname{glg} C \& \operatorname{glg} A(\beta)$ is "off"; consequently the composition speed of glycogen $(\gamma)$ is "slow", though it temporally drops to "no" level. On the other hand, "no" decomposition of glucose takes place in this phase from the same reason as late lag phase above.

Late log phase. Because much glucose was consumed in the previous phase, its uptake speed is going to be slow down. Accordingly, for the phosphate flow in PTS, the input speed of phosphate from PEP becomes faster than the output speed to G6P, causing EIIAGlc phosphorylation "yes" and cAMP level "high". As a result, $g l g C \& g l g A$ activation $(\beta)$ turns "on". In addition, because, in the early half of this phase, FDP is in a high level, $g l g C$ \& $g l g A$ activation $(\alpha)$ is also turned "on". Hence, both $\alpha$ and $\beta$ regulations are working. In this case, according to Table 1, glycogen composition $(\gamma)$ should be marked at "fast" speed. Accompanying with decreasing glucose amount, FDP concentration drops later in this phase, that is "high $\rightarrow$ low", resuling in $g \lg C \& g \lg A$ activation $(\alpha)$ as "on $\rightarrow$ off". As a result, in the later part of this phase, the speed of glycogen 
Table 2 Behaviors of key regulators (HPr and ElIA ${ }^{G / c}$ ) adjusting glucose and glycogen utilization in an E. coli.

\begin{tabular}{|c|c|c|c|c|c|}
\hline \multirow[t]{2}{*}{ Regulator } & \multicolumn{2}{|c|}{ Lag phase } & \multicolumn{2}{|c|}{ Log phase } & \multirow[t]{2}{*}{ Stationary phase } \\
\hline & Early & Late & Early & Late & \\
\hline speed of glucose uptake & very slow & slow & very fast & fast & no \\
\hline FDP amount & low & high & low $\rightarrow$ high & high $\rightarrow$ low & no \\
\hline$\alpha(\operatorname{glg} C \& g \lg \mathrm{A}$ activation $)$ & off & on & off $\rightarrow$ on & on $\rightarrow$ off & off \\
\hline EIIA $^{G / C}$ phosphorylation & yes $\rightarrow$ no & no & no & yes & yes \\
\hline (regulated cAMP level) & high $\rightarrow$ low & low & low & high & high \\
\hline$\beta$ (glgC $\&$ glgA activation) & on $\rightarrow$ off & off & off & on & on \\
\hline X(composition) & no & slow & slow & fast $\rightarrow$ slow & no \\
\hline (decomposition) & slow $\rightarrow$ fast & no & no & no & slow \\
\hline (phosphorylation) & yes $\rightarrow$ no & no & no & yes & yes \\
\hline (localization) & pole & cytosol & cytosol & cytosol & pole \\
\hline
\end{tabular}

In this table, the five proliferation phases (e.g. Late lag phase) are corresponding with their processes of experiment and simulation data in Figure 4.

composition $(\gamma)$ changes as "fast $\rightarrow$ slow", because degragation of enzyme needs time. On the other hand, in this phase, HPr is still in cytosol working for PTS, not for glycogenolysis. In all, since "fast" composition and "no" decomposition are conducted, glycogen accumulates quickly in this period.

Stationary phase. In this period, because extracellular glucose has been totally consumed off, the speed of glycogen is marked as "no" despite the "on" state of $\operatorname{glg} C \& \operatorname{glg} A$ activation $(\beta)$. Hence there is "no" glycogen composition $(\gamma)$. Because of the inactive PTS and the high amount glycogen, (P)HPr is concentrated at the "poles", decomposing glycogen $(\gamma)$ in a "slow speed" for long survival of cells.

\section{Conclusion}

Some works focus on modeling glycolysis, pentose phosphate pathway, TCA cycle etc. [12,26,27], and some focus on the calculation of PTS performance with a protein mass balance theory method [13,34]. And also some of them set up ODE models by combining PTS into their glycolysis pathways $[26,27]$. But none of them take the glycogen metabolic pathway into account. In this work we firstly integrated general mass action based glycogen metabolism model, mass balance theory based PTS model, and ODE model of glycolysis and PP pathway into a computational model with HFPN.

By applying this model, basic regulators for $E$. coli to utilize extracellular glucose and intracellular glycogen were identified. That is, (P)HPr not only works as a member of PTS enzymes but also functions to realize different catalyzing speeds of glycogen by its phosphorylation state combined with GlgP. Actually, phosphorylation state of $(\mathrm{P}) \mathrm{HPr}$ is controlled by the phosphate flux speed influx and outflux of PTS, and this flux speed is controlled by gene expression, subcellular localization, and metabolite concentration (glucose, PEP, FDP). HPr and EIIA ${ }^{G l c}$ are considered to be key roles among these regulators during the utilization of glycogen and glucose by E. coli.

Based on the model with regulatory systems in this work, we provided a systematic view of glucose and glycogen utilization by $E$. coli. This confirms our previous conclusion that glycogen plays an important role as a primary carbon source in lag phase [1].

\section{Methods}

Before achieved our final version integrated HFPN model (Model-4, see Additional file 4[36]), which shows the dynamic time course model of extended central metabolism pathway (glycolysis, pentose phosphate (PP) pathway, glycogen metabolic pathway, PTS and regulators), we have set up 3 preliminary models: a model of glycolysis and PP pathways (Model-1, see Additional file $1[36])$, transplanted from ODE models; a model of PTS (Model-2, see Additional file 2[36]), according to mass balance theory based PTS models; and a combined model of glycolysis, PP pathway and PTS (Model-3, see Additional file 3[36]). By applying regulatory systems and glycogen metabolism network to Model-3, a dynamic HFPN model of central metabolism is settled as Model-4. The method of biological experiment was explained in our previous paper [1], of which the current study is the continuation. More detail modeling approach is in Additional file 6[36].

\section{Additional material}

Additional file 1: Model-1. Cell Illustrator 4.0 file of HFPN model of central metabolism pathway (glycolysis and pentose phosphate pathway), which can be download from URL [36].

Additional file 2: Model-2. Cell Illustrator 4.0 file of HFPN model of PTS, which can be download from URL [36].

Additional file 3: Model-3. Cell Illustrator 4.0 file of HFPN model of combination of central metabolism pathway and PTS, which can be download from URL [36]. 
Additional file 4: Model-4. Cell Illustrator 4.0 file of dynamic HFPN model of central metabolism pathway, PTS and glycogen metabolism with the regulatory mechanisms, which can be download from URL [36].

Additional file 5: Figure S. Additional Figures, which also can be download from URL [36].

Additional file 6: Methods. Detail Methods, which also can be download from URL [36].

Additional file 7: Table S. Additional Tables, which also can be download from URL [36].

\section{List of abbreviation used}

Hybrid functional Petri net (HFPN), ordinary differential equation (ODE), pentose phosphate pathway (PP pathway), metabolite level (M-level), molecule localization level (L-level), phosphate flux level (F-level), protein level(P-level), gene expression level (G-level), phosphorylated enzyme E (PE) (e.g. PHPr), chemical Binding (A:B) (e.g. HPr::GlgP), phosphoenolpyruvate: sugar phosphotransferase system (PTS) glucose 6-phosphate (G6P), glucose 1-phosphate (G1P), ADP-glucose (ADPG), fructose 6-phosphate (F6P), fructose-1,6-bisphosphate (FDP), phosphoenolpyruvate (PEP), pyruvate (PYR), D-glyceraldehyde 3-phosphate (GAP), dihydroxyacetone phosphate (DHAP), 1,3-bisphospho-D-glycerate (PGP), 3-phospho-D-glycerate (3PG), 2-phosphoD-glycerate (2PG), 6-Phosphogluconolactone (6PG), ribulose 5-phos- phate (Ribu5P), ribulose 5-phosphate (R5P), xylulose 5-phosphate (Xyl5P), erythrose 4-phosphate (E4P), sedoheptulose-7-phosphate (S7P), ptsHIcrr (PTS enzymes operon), glgBXCAP (glycogen enzymes operon).

\section{Competing interests}

The authors declare that they have no competing interests.

\section{Authors' contributions}

Z. Tian performed the study of modeling and data analysis. A. Fauré and $\mathrm{H}$. Matsuno worked on this study with giving comments to Z. Tian. H. Mori worked on biology knowledge support. All authors approved the final manuscript.

\section{Acknowledgements}

We would like to thank Dr. Yukako Tohsato for her helpful suggestions in the algorithms and modeling given in this paper. This work was supported by Grant-in-Aid for Scientific Research on Innovative Areas "Synthetic Biology" (24119509) and Grant-in-Aid for Scientific Research (A) (25250028) from JSPS.

\section{Declarations}

Publication of this article was funded by Grant-in-Aid for Scientific Research on Innovative Areas "Synthetic Biology" (24119509) from JSPS.

This article has been published as part of BMC Systems Biology Volume 7

Supplement 6, 2013: Selected articles from the 24th International Conference on Genome Informatics (GIW2013). The full contents of the supplement are available online at http://www.biomedcentral.com/bmcsystbiol/supplements/7/S6.

\section{Authors' details}

'Graduate School of Science and Engineering, Yamaguchi University, 1677-1 Yoshida, 753-8512 Yamaguchi-shi, Yamaguchi, Japan. ${ }^{2}$ Graduate School of Biological Sciences, Nara Institute of Science and Technology, 8916-5 Takayama, 630-0101 Ikoma, Nara, Japan.

Published: 13 December 2013

\section{References}

1. Yamamotoya T, Dose $\mathrm{H}$, Tian Z, Fauré A, Toya Y, Honma M, Igarashi K, Nakahigashi K, Soga T, Mori H, Matsuno H: Glycogen is the primary source of glucose during the lag phase of e. coli proliferation. Biochimica et Biophysica Acta - Proteins and Proteomics 2012, 1824(12):1442-1448.

2. Wilson WA, Roach PJ, Montero M, Baroja-Fernández E, Muõz FJ, Eydallin G, Viale AM, Pozueta-Romero J: Regulation of glycogen metabolism in yeast and bacteria. FEMS Microbiology Reviews 2010, 34(6):952-985.
3. Kotrba P, Inui M, Yukawa H: Bacterial phosphotransferase system (pts) in carbohydrate uptake and control of carbon metabolism. Journal of Bioscience and Bioengineering 2001, 92(6):502-517.

4. Edwards AN, Patterson-Fortin LM, Vakulskas CA, Mercante JW, Potrykus K, Vinella D, Camacho MI, Fields JA, Thompson SA, Georgellis D, Cashel M, Babitzke $P$, Romeo T: Circuitry linking the csr and stringent response global regulatory systems. Molecular Microbiology 2011, 80(6):1561-1580.

5. Baker CS, Morozov I, Suzuki K, Romeo T, Babitzke P: CsrA regulates glycogen biosynthesis by preventing translation of glgC in escherichia coli. Molecular Microbiology 2002, 44(6):1599-1610.

6. Shimada T, Yamamoto $K$, Ishihama A: Novel members of the cra regulon involved in carbon metabolism in escherichia coli. Journal of Bacteriology 2011, 193(3):649-659

7. Vinuselvi P, Kim MK, Lee SK, Ghim CM: Rewiring carbon catabolite repression for microbial cell factory. BMB reports 2012, 45(2):59-70.

8. Deutscher J, Francke C, Postma PW: How phosphotransferase systemrelated protein phosphorylation regulates carbohydrate metabolism in bacteria. Microbiology and Molecular Biology Reviews 2006, 70(4):939-1031.

9. Francke C, Postma PW, Westerhoff HV, Blom JG, Peletier MA: Why the phosphotransferase system of escherichia coli escapes diffusion limitation. Biophysical Journal 2003, 85(1):612-622.

10. Kawamukai M, Utsumi R, Sakai H, Komano T: Molecular cloning and sequencing of the glycogen phosphorylase gene from escherichia coli. FEBS Letters 1989, 243(2):193-198.

11. Nagasaki M, Yamaguchi R, Yoshida R, Imoto S, Doi A, Tamada Y, Matsuno H, Miyano S, Higuchi T: Genomic data assimilation for estimating hybrid functional petri net from time-course gene expression data. Genome Informatics Series 2006, 17(1):46.

12. Chassagnole C, Noisommit-Rizzi N, Schmid JW, Mauch K, Reuss M: Dynamic modeling of the central carbon metabolism of escherichia coli. Biotechnology and bioengineering 2002, 79(1):53-73.

13. Rohwer JM, Meadow ND, Roseman S, Westerhoff HV, Postma PW: Understanding glucose transport by the bacterial Phosphoenolpyruvate: Glycose phosphotransferase system on the basis of kinetic measurements in vitro. Journal of Biological Chemistry 2000, 275(45):34909-34921.

14. Sondej M, Badawi P, Lewis MS, Briggs MC, Jaffe H, Peterkofsky A: High affinity binding and allosteric regulation of escherichia coli glycogen phosphorylase by the histidine phosphocarrier protein, HPr. Journal of Biological Chemistry 1997, 272(42):26511-26521.

15. Koo BM, Seok YJ: Regulation of glycogen concentration by the histidinecontaining phosphocarrier protein $\mathrm{HPr}$ in escherichia coli. Journal of Microbiology -Seoul 2001, 39(1):24-30

16. Seok YJ, Koo BM, Sondej M, Peterkofsky A: Regulation of e. coli glycogen phosphorylase activity by HPr. Journal of molecular microbiology and biotechnology 2001, 3(3):385-394.

17. Romeo T, Preiss J: Genetic regulation of glycogen biosynthesis in escherichia coli: in vitro effects of cyclic AMP and guanosine $5^{\prime}$-diphosphate $3^{\prime}$-diphosphate and analysis of in vivo transcripts. Journal of Bacteriology 1989, 171(5):2773-2782.

18. Gabor E, Göhler AK, Kosfeld A, Staab A, Kremling A, Jahreis K: The phosphoenolpyruvate-dependent glucose-phosphotransferase system from escherichia coli $\mathrm{k}-12$ as the center of a network regulating carbohydrate flux in the cell. European journal of cell biology 2011, 90(9):711-720.

19. Patel HV, Vyas KA, Savtchenko R, Roseman S: The Monomer/Dimer transition of enzyme i of the escherichia coli phosphotransferase system. Journal of Biological Chemistry 2006, 281(26):17570-17578.

20. Lopian L, Elisha Y, Nussbaum-Shochat A, Amster-Choder O: Spatial and temporal organization of the e. coli pts components. The EMBO Journal 2010, 29(21):3630-3645

21. Kohn KW, Aladjem MI, Weinstein JN, Pommier Y: Molecular interaction maps of bioregulatory networks: A general rubric for systems biology. Molecular Biology of the Cell 2006, 17(1):1-13.

22. GenoBase: Results of Protein Localization References. [http://ecolinaist.jp/ GB8-dev/GFP/gfp_result.jsp?fword=JW3643].

23. Traxler MF, Summers SM, Nguyen HT, Zacharia VM, Hightower GA, Smith JT, Conway T: The global, ppGpp-mediated stringent response to amino acid starvation in escherichia coli. Molecular Microbiology 2008, 68(5):1128-1148 
24. Montero M, Almagro G, Eydallin G, Viale AM, Munõz FJ, Bahaji A, Li J, Rahimpour M, Baroja-Fernández E, Pozueta-Romero J: Escherichia coli glycogen genes are organized in a single glgBXCAP transcriptional unit possessing an alternative suboperonic promoter within glgC that directs glgAP expression. Biochemical Journal 2011, 433(1):107-117.

25. Vieira APdA, da Silva MAP, Langone MAP: Biodiesel production via esterification reactions catalyzed by lipase. Latin American applied research 2006, 36(4):283-288.

26. Usuda $Y$, Nishio $Y$, Iwatani S, Van Dien SJ, Imaizumi A, Shimbo K, Kageyama N, Iwahata D, Miyano H, Matsui K: Dynamic modeling of escherichia coli metabolic and regulatory systems for amino-acid production. Journal of Biotechnology 2010, 147(1):17-30.

27. Kadir T, Mannan A, Kierzek A, McFadden J, Shimizu K: Modeling and simulation of the main metabolism in escherichia coli and its several single-gene knockout mutants with experimental verification. Microbial Cell Factories 2010, 9(1):88.

28. BioModels: [http://www.ebi.ac.uk/biomodels-main/BIOMD0000000051]

29. Baldazzi $V$, Ropers D, Markowicz Y, Kahn D, Geiselmann J, de Jong H: The carbon assimilation network in escherichia coli is densely connected and largely sign-determined by directions of metabolic fluxes. PLOS computational biology 2010, 6(6):1000812.

30. Tohsato $Y$, Ikuta K, Shionoya A, Mazaki Y, Ito M: Parameter optimization and sensitivity analysis for large kinetic models using a real-coded genetic algorithm. Gene 2013, 518(1):84-90.

31. Schomburg I, Chang A, Ebeling C, Gremse M, Heldt AC, Huhn G: Schomburg D: BRENDA, the enzyme database: updates and major new developments. Nucleic Acids Research 2004, 32(suppl 1):431-433.

32. Wittig U, Kania R, Golebiewski M, Rey M, Shi L, Jong L, Algaa E, Weidemann A, Sauer-Danzwith H, Mir S, Krebs O, Bittkowski M, Wetsch E, Rojas I, Muller W: SABIO-RK-database for biochemical reaction kinetics. Nucleic Acids Research 2011, 40(D1):790-796.

33. Li C, Donizelli M, Rodriguez N, Dharuri H, Endler L, Chelliah V, Li L, He E, Henry A, Stefan MI, Snoep JL, Hucka M, Novère NL, Laibe C: BioModels database: An enhanced, curated and annotated resource for published quantitative kinetic models. BMC Systems Biology 2010, 4(1):92

34. Rodríguez JV, Kaandorp JA, Dobrzyñski M, Blom JG: Spatial stochastic modelling of the phosphoenolpyruvate-dependent phosphotransferase (pts) pathway in escherichia coli. Bioinformatics 2006, 22(15):1895-1901.

35. Nagasaki M, Saito A, Jeong E, Li C, Kojima K, Ikeda E, Miyano S: Cell illustrator 4.0: A computational platform for systems biology. In Silico Biology 2010, 10(1):5-26.

36. [http://genome.ib.sci.yamaguchi-u.ac.jp/etchp/GIW2013/], Additional Files of this Paper.

37. Wikipedia: Pearson Product-moment Correlation Coefficient. [http://en wikipedia.org/wiki/Pearson_product-moment_correlation_coefficient].

doi:10.1186/1752-0509-7-S6-S1

Cite this article as: Tian et al:: Identification of key regulators in glycogen utilization in E. coli based on the simulations from a hybrid functional Petri net model. BMC Systems Biology 2013 7(Suppl 6):S1.

\section{Submit your next manuscript to BioMed Central and take full advantage of:}

- Convenient online submission

- Thorough peer review

- No space constraints or color figure charges

- Immediate publication on acceptance

- Inclusion in PubMed, CAS, Scopus and Google Scholar

- Research which is freely available for redistribution 